\title{
Effectiveness of Chemoradiotherapy for Metachronous Esophageal Squamous Cell Carcinoma
}

\author{
Yugo Suzukia $^{a}$ Daisuke Kikuchi ${ }^{a}$ Shu Hoteya ${ }^{a}$ Takayuki Okamura ${ }^{a}$ \\ Yorinari Ochiai $^{a}$ Junnosuke Hayasaka ${ }^{a}$ Nobuhiro Dan ${ }^{a}$ Yutaka Mitsunaga ${ }^{a}$ \\ Masami Tanaka ${ }^{a}$ Hiroyuki Odagiria Kosuke Nomura ${ }^{a}$ Satoshi Yamashita ${ }^{a}$ \\ Akira Matsui $^{a}$ Toshiro lizukab \\ aDepartment of Gastroenterology, Toranomon Hospital, Tokyo, Japan; bepartment of Gastroenterology, \\ Tokyo Metropolitan Cancer and infectious Diseases Center Komagome Hospital, Tokyo, Japan
}

\section{Keywords}

Chemoradiotherapy - Metachronous esophageal squamous cell carcinoma $\cdot$ Multiple Lugol-voiding lesions

\begin{abstract}
Introduction: Multiple Lugol-voiding lesions (LVLs) in the esophagus increase the risk of synchronous and metachronous development of esophageal squamous cell carcinoma (ESCC). Chemoradiotherapy (CRT) following endoscopic submucosal dissection (ESD) may reduce the incidence of metachronous ESCC, but few studies have investigated this. Therefore, we retrospectively examined the effect of CRT on metachronous ESCC and multiple esophageal dysplasias visible as multiple LVLs. Methods: This study reviewed 146 patients who underwent esophageal ESD and were determined pathologically to have noncurative resection. They were divided into 2 groups: those who received additional CRT (CRT group; $n=64$ ) and those without additional treatment (control group; $n=82$ ). Incidence of metachronous ESCC was analyzed using propensity scores to adjust for patient characteristics. The number of multiple LVLs was also examined. $\boldsymbol{R e}$ sults: The CRT group was significantly younger than the
\end{abstract}

\begin{tabular}{ll}
\hline karger@karger.com & $\begin{array}{l}\text { (C) } 2020 \text { The Author(s) } \\
\text { Published by S. Karger AG, Basel } \\
\text { www.karger.com/dig }\end{array}$ \\
Karger & $\begin{array}{l}\text { This is an Open Access article licensed under the Creative Commons } \\
\text { Attribution-NonCommercial-4.0 International License (CC BY-NC) } \\
\text { (http://www.karger.com/Services/OpenAccessLicense), applicable to } \\
\text { the online version of the article only. Usage and distribution for com- } \\
\text { mercial purposes requires written permission. }\end{array}$
\end{tabular}

control group (mean 66.6 vs. 70.6 years, $p=0.011$ ), had significantly deeper tumor invasion $(p=0.013)$, and had a significantly higher rate of lymphovascular invasion (47.8 vs. $12.2 \%, p<0.001)$. The CRT group also had a significantly higher improvement rate of multiple LVLs ( $58.1 \mathrm{vs.} 2.0 \%, p<0.001$ ). The LVLs after CRT had a distinctive irregular crack-shaped appearance. Metachronous ESCC was found in 7 patients (10.9\%) in the CRT group and in 17 patients $(20.7 \%)$ in the control group $(p=0.113)$. In propensity score-adjusted logistic regression analysis, the odds ratio for metachronous ESCC in the CRT group was $0.316(p=0.023)$. The occurrence rate was significantly lower in the CRT group than in the control group. Discussion/Conclusion: CRT may be effective in preventing metachronous ESCC. 02020 The Author(s) Published by S. Karger AG, Basel

\section{Introduction}

The outcome of endoscopic resection is favorable for pT1a-epithelium/lamina propria mucosae esophageal cancer $[1,2]$. However, additional treatment such as surgery and chemoradiotherapy (CRT) has been recom-

\footnotetext{
Yugo Suzuki

Department of Gastroenterology, Toranomon Hospital

2-2-2 Toranomon, Minato-ku

Tokyo 105-8470 (Japan)

yugo-suzuki@nms.ac.jp
} 

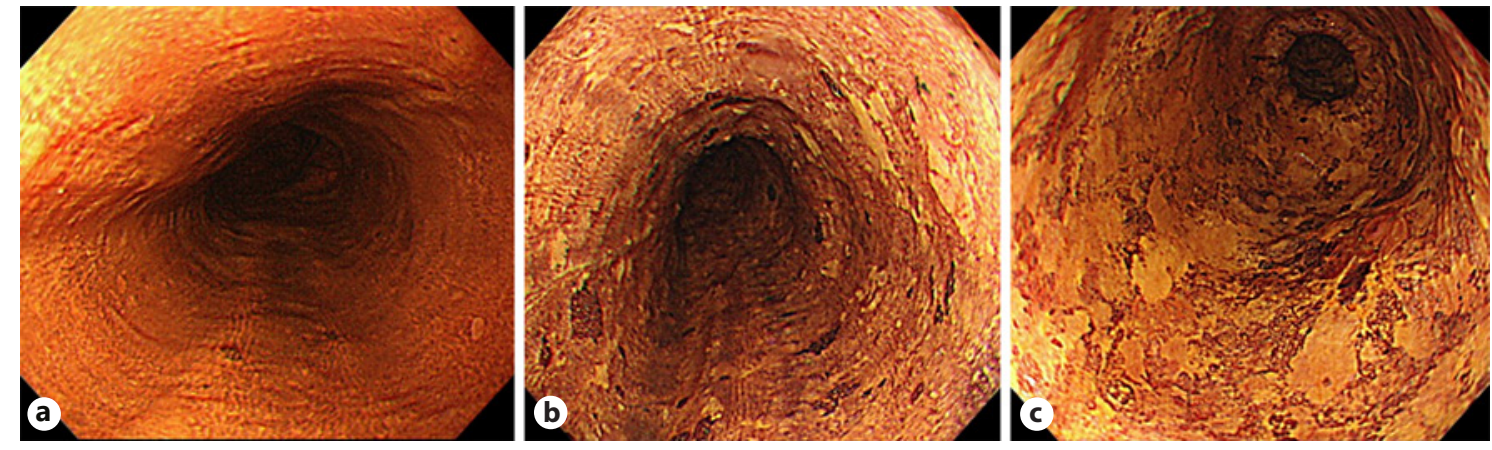

Fig. 1. The background esophageal mucosa is classified according to 3 grades based on the number of LVLs identified by chromoendoscopy: no LVLs (a); several LVLs $(\leq 10)(\mathbf{b})$; and many LVLs (>10) (c). LVLs, Lugol-voiding lesions.

mended for esophageal squamous cell carcinoma (ESCC) invading deeper than the muscularis mucosae (MM) [3, 4]. Appreciable advances in the radiation technology now afford comparable long-term outcomes between CRT and radical esophagectomy in patients with superficial ESCC [5]. However, metachronous esophageal cancer is an important issue to address in patients who have received organ-preservation treatment for esophageal cancer.

Synchronous and metachronous development of squamous cell carcinoma (SCC) in the stratified epithelium of the upper digestive tract, including the esophagus and laryngopharynx, is a phenomenon that has been referred to as field cancerization [6]. Multiple dysplastic lesions in the esophagus also increase the risk of multiple ESCC $[7,8]$. The occurrence rate of metachronous SCC after endoscopic resection was reported to be $13-14.6 \%$ $[9,10]$ and $22 \%$ in patients with stage I/II ESCC who received CRT [11]. The radiation field is typically generous to account for subclinical extension of the cancer along the esophagus to the regional lymph nodes and thus covers a large area of the esophagus. This coverage may reduce the incidence of metachronous multiple esophageal cancer compared with that in patients followed up without treatment, but few studies have investigated this. In this study, we examined the effectiveness of CRT on metachronous ESCC and multiple esophageal dysplasias visible as multiple Lugol-voiding lesions (LVLs).

\section{Materials and Methods}

Patients and Design

This study reviewed 767 patients with ESCC who underwent endoscopic submucosal dissection (ESD) at Toranomon Hospital, Tokyo, Japan, between January 1, 2010, and April 1, 2019. All ESD specimens were assessed pathologically based on the Japanese Classification of Esophageal Cancer [12], in which complete resection ( $\mathrm{R} 0$ resection) is defined as en bloc resection with margins free of tumor. However, findings such as depth of invasion and lymphovascular infiltration are not included in this definition. Curative resection was defined based on the Guidelines for Diagnosis and Treatment of Carcinoma of the Esophagus, with the resected specimen meeting the requirements for R0 resection and showing no invasion into the lymph duct or venous duct or into MM or deeper [3]. Any additional treatment provided, such as surgical treatment or CRT, was recommended for noncurative resections. In this study, cases of MM without lymphovascular invasion were defined as the noncurative resection, and patients were well informed about the risks and benefits and decided whether to treat at their will.

We retrospectively analyzed those patients who were determined pathologically to have noncurative resection, excluding those who had a history of preceding chemotherapy and/or radiation therapy or those who had received additional surgical treatment and additional chemotherapy or radiation therapy alone. A total of 146 consecutive patients were divided into 2 groups: those who received additional CRT (CRT group; $n=82$ ) and those who did not receive additional treatment (control group; $n=64$ ). Their clinical information and endoscopic findings were then collected and compared. All patients were informed about the importance of the abstinence during hospitalization for treatment. We surveyed drinking status by self-reporting and instructed patients not to drink. Clinical findings during follow-up were also analyzed; noncurative ESD cases basically underwent endoscopy, computed tomography, and endoscopic ultrasonography once every 6 months for at least 5 years after ESD, and thereafter, they undergo endoscopy twice a year and computed tomography and endoscopic ultrasonography once a year. Metachronous ESCC was defined as ESCC detected after ESD, and the time to incidence of metachronous ESCC was defined as the interval between the day ESD was performed and the day a new lesion was detected.

This study was performed in accordance with the ethical standards laid down in the 1964 Declaration of Helsinki and its later amendments. The study was approved by the ethics committee of our hospital, and written informed consent was obtained from all patients to undergo the proposed procedure. 
Table 1. Clinicopathological characteristics of the patients

\begin{tabular}{|c|c|c|c|}
\hline & CRT $(n=64)$ & Control $(n=82)$ & $p$ value \\
\hline Age, years, mean \pm SD & $66.6 \pm 9.03$ & $70.6 \pm 9.399$ & $0.011^{*}$ \\
\hline Gender, male:female, \% & $59: 5,92.2$ & $74: 8,90.2$ & 0.682 \\
\hline Brinkman index & $863 \pm 799.7$ & $727 \pm 605$ & 0.211 \\
\hline Alcohol consumption, g/day & $72.8 \pm 76.8$ & $53.6 \pm 49.9$ & $0.038^{*}$ \\
\hline \multicolumn{4}{|l|}{ Location, $n(\%)$} \\
\hline $\mathrm{Ce}$ & $3(4.7)$ & $1(12.2)$ & 0.178 \\
\hline Ut & $7(10.9)$ & $17(20.7)$ & \\
\hline Mt & $37(57.8)$ & $38(46.3)$ & \\
\hline $\mathrm{Lt}$ & $17(26.6)$ & $26(31.7)$ & \\
\hline \multicolumn{4}{|l|}{ Morphological type, $n(\%)$} \\
\hline Type 0-I & $5(7.8)$ & $6(7.3)$ & 0.265 \\
\hline Type 0-IIa & $8(12.5)$ & $9(11.0)$ & \\
\hline Type 0-IIb & $2(3.1)$ & $10(12.2)$ & \\
\hline Type $0-$-IIc & $49(76.6)$ & $57(69.5)$ & \\
\hline Tumor size, $\mathrm{mm}$, mean $\pm \mathrm{SD}$ & $30.1 \pm 17.7$ & $32.5 \pm 19.3$ & 0.435 \\
\hline \multicolumn{4}{|l|}{ Depth of invasion, $n(\%)$} \\
\hline $\mathrm{MM}$ & $29(45.3)$ & $58(70.7)$ & $0.013^{*}$ \\
\hline SM1 & $11(17.2)$ & $10(12.2)$ & \\
\hline SM2 & $24(37.5)$ & $14(17.1)$ & \\
\hline Lymphovascular invasion, $n(\%)$ & $30(46.9)$ & $14(12.2)$ & $<0.001^{*}$ \\
\hline Observation period, months, mean $\pm S D$ & $74.1 \pm 34.3$ & $57.4 \pm 31.0$ & $<0.001^{*}$ \\
\hline
\end{tabular}

CRT, chemoradiotherapy; Ce, cervical part of the thoracic esophagus; Lt, lower part of the thoracic esophagus; $\mathrm{MM}$, muscularis mucosae; Mt, middle part of the thoracic esophagus; SD, standard deviation; SM, submucosa; Ut, upper part of the thoracic esophagus. ${ }^{*} p<0.05$.

\section{CRT Regimen}

Within 60 days after ESD, concomitant CRT was started in accordance with the regimen in the JCOG0508 study [13]. The chemotherapy regimen was continuous 5 -fluorouracil (5-FU; $700 \mathrm{mg}$ / $\mathrm{m}^{2} /$ day, days $1-4$ and 29-32) and cisplatin (CDDP; $70 \mathrm{mg} / \mathrm{m}^{2} /$ day, days 1 and 29). The dose of radiotherapy was $41.4 \mathrm{~Gy} / 23 \mathrm{Fr} / 5$ weeks (5 days/week) for cases with a negative resection margin. The toxicity of CRT was retrospectively evaluated in accordance with the National Cancer Institute Common Terminology Criteria for Adverse Events (version 3.0).

\section{Endoscopic Findings}

Location, size, and macroscopic features of each lesion were determined from images obtained by white light endoscopy and chromoendoscopy (1\% Lugol's iodine solution). Diagnoses were obtained retrospectively based on the Japanese Classification of Esophageal Cancer [12] by 2 board-certified fellows of the Japan Gastroenterological Endoscopy Society (JGES). According to the Lugol staining, LVLs were defined as the areas void of the stain. Background esophageal mucosa was also evaluated based on the number of LVLs per endoscopic view, as (A) none, (B) several $(\leq 10)$, or $(\mathrm{C})$ many $(>10)$ (Fig. 1) [7, 14].

\section{Histopathological Assessment of the Resected Specimens}

All ESD specimens were fixed in 10\% formalin, sectioned serially at 2-mm intervals, stained with hematoxylin and eosin, and assessed pathologically based on the Japanese Classification of Esophageal Cancer [12]. Histological type, tumor size, depth of invasion, resection margins, and lymphovascular invasion were eval- uated in each slice by specialized pathologists from the Japanese Society of Pathology.

\section{Statistical Analysis}

Data are presented as the mean \pm SD. Statistical analyses were performed using an unpaired $t$ test, $\chi^{2}$ test, Fisher's exact test, or the Mann-Whitney $U$ test as appropriate. A $p$ value of $<0.05$ was considered significant. All statistical analyses were performed using SPSS version 25 (SPSS IBM statistics; IBM Corp., Armonk, NY, USA).

To adjust for patient characteristics, we calculated propensity scores using logistic regression analysis. Propensity scores were estimated using the following variables: sex, age, alcohol consumption, smoking, lesion location, lesion size, morphological type, tumor depth, and multiple LVLs. Each patient in the CRT group was matched with a patient in the control group with the closest estimated propensity score on the logit scale. C-statistics were calculated, and logistic regression analysis was performed to assess the association between occurrence of metachronous ESCC and CRT with adjustment for propensity scores.

\section{Results}

\section{Clinicopathological Characteristics}

Table 1 shows the patients' clinicopathological characteristics. Three patients received continuous 5-FU (800 


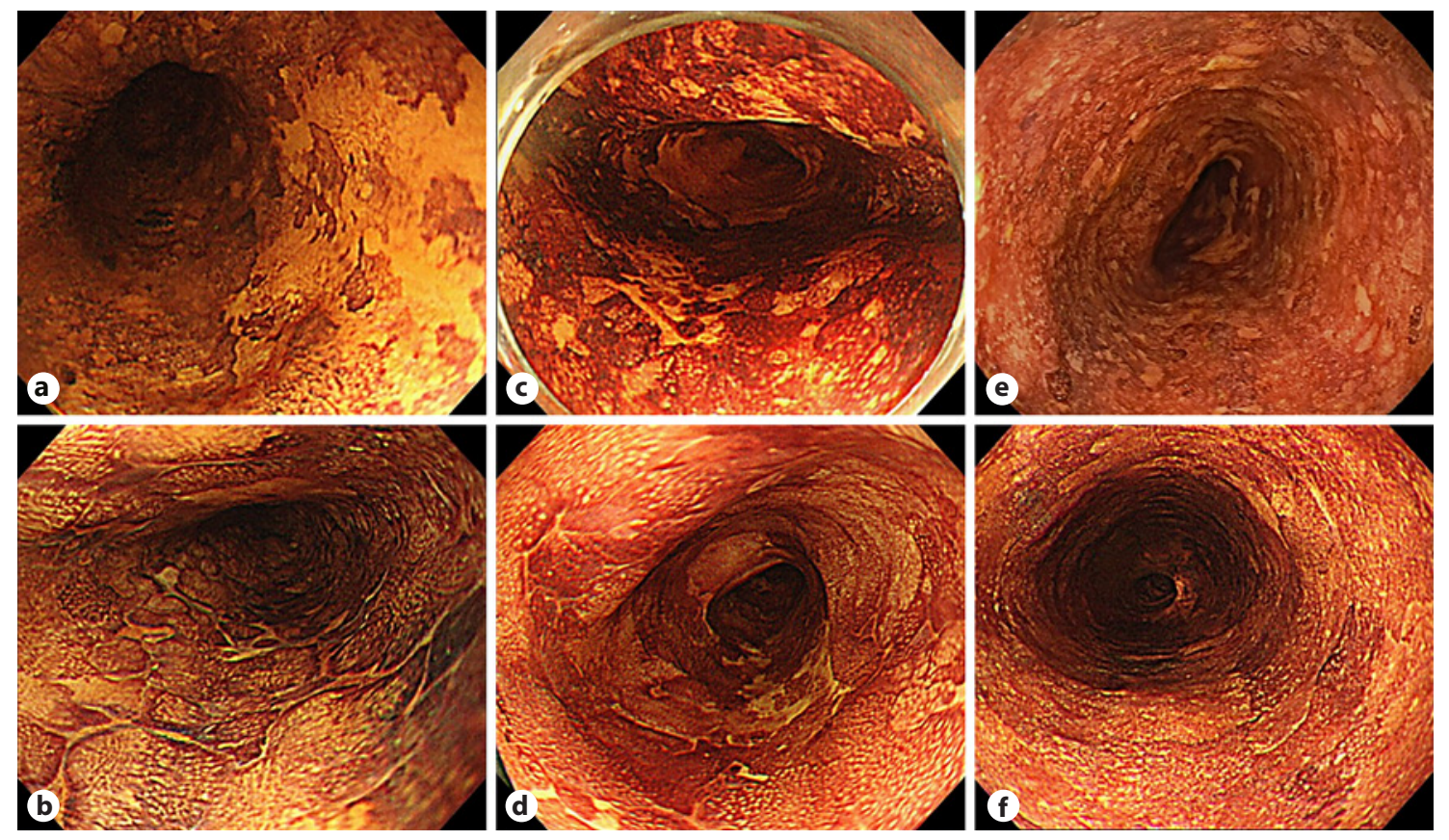

Fig. 2. Chromoendoscopy before ESD and after CRT in 3 cases. Case 1 before ESD (a) and after CRT (b). LVLs after CRT (b) had an irregular crack-shaped appearance, and the number of LVLs decreased. Case 2 before ESD (c) and after CRT (d). LVLs after CRT (d) had an irregular crack-shaped appearance, and the number of LVLs decreased. Case 3 before ESD (e) and after CRT (f). LVLs almost disappeared after CRT. ESD, endoscopic submucosal dissection; CRT, chemoradiotherapy; LVLs, Lugol-voiding lesions.

Table 2. Background esophageal multiple LVLs

\begin{tabular}{cccc}
\hline & $\begin{array}{l}\text { CRT } \\
(n=64)\end{array}$ & $\begin{array}{l}\text { Control } \\
(n=82)\end{array}$ & $p$ value \\
\hline Multiple LVLs before ESD, $n(\%)$ & & \\
A & 0 & 0 & 0.235 \\
B & $36(56.3)$ & $38(46.3)$ & \\
C & $28(43.8)$ & $44(53.7)$ & \\
Multiple LVLs after treatment, $n(\%)$ & & \\
A & $9(20.9)$ & $1(2.0)$ & $<0.001^{*}$ \\
B & $32(74.4)$ & $20(40.0)$ & \\
C & $2(4.7)$ & $29(58.0)$ & \\
Improvement of LVLs, $n(\%)$ & $25 / 43(58.1)$ & $1 / 50(2.0)$ & $<0.001^{*}$ \\
& &
\end{tabular}

CRT, chemoradiotherapy; ESD, endoscopic submucosal dissection; LVLs, Lugol-voiding lesions. ${ }^{*} p<0.05$.

$\mathrm{mg} / \mathrm{m}^{2} /$ day, days $1-5$ and $\left.29-33\right)$ and nedaplatin (800 $\mathrm{mg} / \mathrm{m}^{2} /$ day, days 1 and 29), and 1 patient received lowdose docetaxel (Taxotere; $10 \mathrm{mg} / \mathrm{m}^{2} /$ day, days $1,8,15,22$, 29 , and 36) instead of the 5-FU and CDDP regimen. The dose of radiotherapy was $50.4 \mathrm{~Gy} / 28 \mathrm{Fr} / 5$ weeks (5 days/ week) with a boost at the primary site for the 1 case with a positive resection margin. The male-to-female ratio was 59:5 in the CRT group and 74:8 in the control group. The CRT group was significantly younger than the control group (mean age $66.6 \pm 9.03$ years vs. $70.6 \pm 9.40$ years; $p=0.011)$ and had significantly higher alcohol consumption (72.8 \pm 76.8 vs. $53.6 \pm 49.9 \mathrm{~g} /$ day; $p=0.038)$. There was no significant difference in the Brinkman index between the 2 groups $(863 \pm 799.7$ vs. $727 \pm 605)$.

Lesion analysis revealed that tumor location, morphological type, and tumor size were not significantly different between the 2 groups. However, the CRT group had significantly deeper tumor invasion $(p=0.013)$ and a significantly higher rate of lymphovascular invasion (46.9 vs. $12.2 \%, p<0.001)$. The follow-up period was significantly longer in the CRT group at a mean of $74.1 \pm 34.3$ months compared with $57.4 \pm 31.0$ months in the control group $(p<0.001)$.

Twenty six (49.1\%) of 53 patients in the CRT group who drank $1 \mathrm{U} /$ day (22 $\mathrm{g}$ of ethanol) or more at baseline abstained from alcohol. On the other hand, in the control group, 29 (51.8\%) of 56 patients abstained from alcohol, and there was no significant difference between 2 groups $(p=0.776)$. 
Table 3. Incidence of metachronous ESCC

\begin{tabular}{|c|c|c|c|c|c|c|}
\hline & \multicolumn{3}{|l|}{ All patients } & \multicolumn{3}{|c|}{ Propensity-matched patients } \\
\hline Metachronous ESCC, $n(\%)$ & $7(10.9)$ & $17(20.7)$ & 0.113 & $2(5.1)$ & $8(20.5)$ & $0.042^{*}$ \\
\hline Metachronous ESCC in LVL grade C, $n(\%)$ & $3 / 28(10.7)$ & $16 / 44(36.4)$ & $0.027^{*}$ & & & \\
\hline Time to incidence of metachronous ESCC, months, mean \pm SD & $42.9 \pm 22.5$ & $31.8 \pm 23.1$ & 0.297 & & & \\
\hline
\end{tabular}

CRT, chemoradiotherapy; ESCC, esophageal squamous cell carcinoma; LVLs, Lugol-voiding lesions; SCC, squamous cell carcinoma; $\mathrm{SD}$, standard deviation. $* p<0.05$.

Of 64 patients who received CRT, 33 patients (51.6\%) developed grade 3 or 4 hematologic toxicity: 32 patients (50\%) had grade 3 or 4 leukopenia, 25 patients (39.1\%) had grade 3 or 4 neutropenia, 6 patients $(9.4 \%)$ had grade 3 thrombocytopenia, and 2 patients (3.1\%) had grade 3 febrile neutropenia. Ten patients (15.6\%) developed grade 3 nonhematologic toxicity: 5 patients $(7.8 \%)$ had odynophagia or esophagitis, 2 patients $(3.1 \%)$ had anorexia, 1 patient (1.6\%) developed shingles, and 1 patient (1.6\%) developed internal jugular vein thrombosis due to the central venous catheter.

\section{Multiple Lugol-Voiding Lesions}

Background multiple LVLs according to grade are shown in Table 2. After ESD, 43 patients in the CRT group and 50 patients in the control group were followed up with chromoendoscopy using Lugol staining. Before ESD, there was no significant difference between the 2 groups in the number of grade B or C multiple LVLs, but after ESD, the CRT group showed a significantly higher rate of improvement in the multiple LVL grade than the control group (58.1 vs. $2.0 \%, p<0.001$ ), leading to a significant overall difference in the multiple LVL grade between the 2 groups $(p<0.001)$. In most cases, LVLs after CRT had an irregular crack-shaped appearance (Fig. 2).

\section{Incidence of Metachronous Esophageal Squamous Cell} Carcinoma

The incidence of metachronous ESCC is shown in Table 3. Metachronous ESCC was found in 7 patients $(10.9 \%)$ in the CRT group and in 17 patients $(20.7 \%)$ in the control group $(p=0.113)$. In the CRT group, 2 metachronous lesions were surgically resected and diagnosed as stage I and II ESCC, respectively. The other 22 lesions underwent secondary ESD, resulting in pathologically curative resection. Time to detection of metachronous ESCC was not significantly different between the 2 groups.
The occurrence rate of metachronous ESCC in the patients with grade $\mathrm{C}$ multiple LVLs was lower in the CRT group compared with the control group (10.7 vs. $36.4 \%$, $p=0.027)$ despite the significantly longer follow-up period in the CRT group (74.1 \pm 34.3 vs. $57.4 \pm 31.0$ months, respectively; $p<0.001)$.

In the nonabstainers, 5 patients $(18.5 \%)$ in the CRT group and 9 patients (33.3\%) in the control group developed metachronous ESCC and there was no significant difference. The C-statistic of the propensity score was 0.794 . In the propensity score-adjusted logistic regression analysis, patient distributions were closely balanced between the 2 groups (Table 4), and metachronous ESCC occurrence rate in the CRT group was significantly lower than that in the control group $(p=0.042)$. There was a significant difference even considering the difference of the CRT regimens.

\section{Discussion/Conclusion}

The effectiveness of adding CRT after endoscopic resection in patients with clinical stage I ESCC has been reported as favorable [15-18]. This suggests that metachronous cancer is also an important issue to consider in patients with ESCC, and close endoscopic follow-up is essential when the esophagus is preserved. However, there are few reports on metachronous ESCC after endoscopic resection.

To our knowledge, this study is the first to report improvement in multiple LVLs and the incidence of metachronous ESCC after CRT following ESD compared with patients who did not receive additional treatment. Patient characteristics and pathological findings were significantly different between the CRT and control groups. Specifically, patients were significantly younger and lesions had significantly more lymphovascular invasion and deeper tumor invasion in the CRT group. These findings indicate 
Table 4. Propensity-matched patients' characteristics and background esophageal multiple LVLs

\begin{tabular}{lccc}
\hline & CRT $(n=39)$ & Control $(n=39)$ & $p$ value \\
\hline Age, years, mean \pm SD & $68.7 \pm 8.47$ & $67.8 \pm 9.99$ & 0.67 \\
Gender, male:female, \% & $36: 3,92.3$ & $34: 5,87.2$ & 0.455 \\
Brinkman index & $835 \pm 841$ & $831 \pm 654$ & 0.978 \\
Alcohol consumption, g/day & $65.1 \pm 43.0$ & $68.2 \pm 56.3$ & 0.789 \\
Location, $n(\%)$ & & & \\
Ce & $1(2.6)$ & $1(2.6)$ & 0.881 \\
Ut & $5(12.8)$ & $3(7.7)$ & \\
Mt & $22(56.4)$ & $22(56.4)$ & \\
Lt & $11(28.2)$ & $13(33.3)$ & \\
Morphological type, $n(\%)$ & & & \\
Type 0-I & $3(7.7)$ & $3(7.7)$ & 0.975 \\
Type 0-IIa & $5(12.8)$ & $5(12.8)$ & \\
Type 0-IIb & $2(5.1)$ & $3(7.7)$ & \\
Type 0-IIc & $29(74.4)$ & $28(71.8)$ & \\
Tumor size, mm, mean \pm SD & $32.6 \pm 17.8$ & $32.0 \pm 17.9$ & 0.93 \\
Depth of invasion, $n(\%)$ & $20(51.3)$ & $23(59.0)$ & 0.727 \\
MM & $7(17.9)$ & $7(17.9)$ & \\
SM1 & $12(30.8)$ & $9(23.1)$ & \\
SM2 & $13(33.3)$ & $12(30.8)$ & 0.808 \\
Lymphovascular invasion, $n(\%)$ & & & \\
Multiple LVLs before ESD, $n(\%)$ & 0 & 0 & 0.821 \\
A & $19(48.7)$ & $20(51.3)$ & \\
B & $20(51.3)$ & $19(48.7)$ & \\
C & & & \\
& & & \\
\end{tabular}

CRT, chemoradiotherapy; MM, muscularis mucosae; SM, submucosa; Ce, cervical part of the thoracic esophagus; Lt, lower part of the thoracic esophagus; LVLs, Lugolvoiding lesions; Mt, middle part of the thoracic esophagus; Ut, upper part of the thoracic esophagus. ${ }^{*} p<0.05$. that clinicians should decide on additional treatment after noncurative ESD based on the patient's background and the risk of metastasis estimated from lesional factors.

An association between multiple LVLs and metachronous ESCC has been reported [7-10, 14, 19], and the presence of multiple LVLs is considered a risk factor for the development of ESCC. In this study, the improvement ratio of multiple LVLs was significantly higher in the CRT group than in the control group, although there was no significant difference between the 2 groups in LVLs in the background mucosa. This suggests that improvement of multiple LVLs may help to reduce the incidence of metachronous ESCC. In many cases in the CRT group after treatment, LVLs had an irregular crack-shaped appearance, which was probably due to the effect of the radiation therapy. In this study, we counted consecutive Lugolvoiding areas as one in the lesion of the irregular crackshaped LVLs, and the area of LVLs decreased due to this change in shape. This may be considered a distinctive finding and reflects the lower incidence of metachronous ESCC in the CRT group.

Effectiveness of Chemoradiotherapy for the Metachronous Esophageal Cancer
Concerning metachronous SCC, in addition to the multiple LVLs, it has been found that alcohol consumption, cigarette smoking, and genetic factors such as alcohol dehydrogenase (ADH)-1B and $\mathrm{ADH} 2$ are associated with the development of metachronous ESCC [7, 20-23]. In our study, the level of alcohol consumption was significantly higher in the CRT group. This was considered to be an important confounding factor considering that the incidence of metachronous ESCC is the focus of this study. For this reason, we calculated propensity scores using logistic regression analysis to adjust for these factors.

We observed a significantly lower incidence of metachronous ESCC in the CRT group, suggesting that CRT may be effective in preventing metachronous ESCC after ESD. This is reflected in the improvement seen in multiple LVLs. Furthermore, the difference in incidence between the 2 groups was remarkable in patients with grade $\mathrm{C}$ multiple LVLs. These findings may be useful in treatment selection after noncurative ESD.

Alcohol abstinence decreased the risk of developing metachronous multiple SCC of the esophagus [7]. In our 
study, about half of patients in both groups were able to abstain from alcohol. Further study considering the abstinence will be required.

In this study, all adverse events by CRT were transient and patients had recovered at discharge. The adverse events by CRT following the ESD were considered to be acceptable for treatment.

This study has some limitations. First, it was a singlecenter study with a small sample size and retrospective design. Second, the baseline characteristics were different between the 2 groups and we calculated propensity scores using logistic regression analysis to adjust for this. Third, we did not consider long-term outcomes. A large-scale prospective randomized controlled trial is needed to verify the efficacy of CRT on metachronous ESCC.

In conclusion, the results of this study suggest that CRT following ESD for ESCC reduces the incidence of metachronous ESCC. Confirmation of these results is warranted in a prospective study.

\section{Statement of Ethics}

This study was approved by the Institutional Review Board of Toranomon Hospital (Tokyo, Japan; Approval No. 1970), and the study was conducted in accordance with Helsinki Declaration
(1964, and later versions). This study is a retrospective observational study and does not necessarily require written informed consent because it is a study that uses only information such as medical records without using samples. By disclosing information related to clinical research on our website, we will provide the subject with the opportunity to withdraw from using their own information.

\section{Conflict of Interest Statement}

Yugo Suzuki, Daisuke Kikuchi, Shu Hoteya, Takayuki Okamura, Yorinari Ochiai, Junnosuke Hayasaka, Nobuhiro Dan, Yutaka Mitsunaga, Masami Tanaka, Hiroyuki Odagiri, Kosuke Nomura, Satoshi Yamashita, Akira Matsui, and Toshiro Iizuka declare that they have no conflicts of interest.

\section{Funding Sources}

The authors did not receive any funding.

\section{Author Contributions}

Y.S. and T.I.: conceptualization; Y.S. and T.I.: methodology; all authors: investigation; Y.S.: writing original draft; Y.S., D.K., and T.I.: writing, reviewing, and editing; T.I.: supervision. All authors approved the final version of the manuscript.

\section{References}

1 Katada C, Muto M, Momma K, Arima M, Tajiri $\mathrm{H}$, Kanamaru C, et al. Clinical outcome after endoscopic mucosal resection for esophageal squamous cell carcinoma invading the muscularis mucosae: a multicenter retrospective cohort study. Endoscopy. 2007;39(9): 779-83.

2 Yamashina T, Ishihara R, Nagai K, Matsuura $\mathrm{N}$, Matsui $\mathrm{F}$, Ito $\mathrm{T}$, et al. Long-term outcome and metastatic risk after endoscopic resection of superficial esophageal squamous cell carcinoma. Am J Gastroenterol. 2013;108(4):54451.

3 Kuwano H, Nishimura Y, Oyama T, Kato H, Kitagawa Y, Kusano M, et al. Guidelines for Diagnosis and Treatment of Carcinoma of the Esophagus April 2012 edited by the Japan Esophageal Society. Esophagus. 2015;12:130.

4 Tanaka T, Matono S, Mori N, Shirouzu K, Fujita $\mathrm{H}$. T1 squamous cell carcinoma of the esophagus: long-term outcomes and prognostic factors after esophagectomy. Ann Surg Oncol. 2014;21(3):932-8.

5 Yamamoto S, Ishihara R, Motoori M, Kawaguchi Y, Uedo N, Takeuchi Y, et al. Comparison between definitive chemoradiotherapy and esophagectomy in patients with clinical stage I esophageal squamous cell carcinoma. Am J Gastroenterol. 2011;106 (6): 1048-54.

6 Slaughter DP, Southwick HW, Smejkal W. Field cancerization in oral stratified squamous epithelium; clinical implications of multicentric origin. Cancer. 1953;6(5): 963-8.

7 Katada C, Yokoyama T, Yano T, Kaneko K, Oda I, Shimizu Y, et al. Alcohol consumption and multiple dysplastic lesions increase risk of squamous cell carcinoma in the esophagus, head, and neck. Gastroenterology. 2016; 151(5):860-e7.

8 Urabe Y, Kagemoto K, Nakamura K, Mizumoto T, Sanomura Y, Oka S, et al. Construction of a risk model for the development of metachronous squamous cell carcinoma after endoscopic resection of esopahageal squamous cell carcinoma. Esophagus. 2019;16(2): $141-6$.

9 Shimizu Y, Tukagoshi H, Fujita M, Hosokawa M, Kato M, Asaka M. Metachronous squamous cell carcinoma of the esophagus arising after endoscopic mucosal resection. Gastrointest Endosc. 2001;54(2):190-4..

10 Urabe Y, Hiyama T, Tanaka S, Oka S, Yoshihara M, Arihiro K, et al. Metachronous multiple esophageal squamous cell carcinomas and Lugol-voiding lesions after endoscopic mucosal resection. Endoscopy. 2009;41(4): 304-9.

11 Kumabe A, Fukada J, Kota R, Koike N, Shiraishi Y, Seki S, et al. Long-term results of concurrent chemoradiotherapy with daily-lowdose continuous infusion of 5-fluorouracil and cisplatin (LDFP) for stage I-II esophageal carcinoma. Dis Esophagus. 2018;31(4).

12 Japan Esophageal Society. Japanese Classification of Esophageal Cancer, 11th Edition: part I. Esophagus. 2017;14(1):1-36.

13 Kurokawa Y, Muto M, Minashi K, Boku N, Fukuda H. A phase II trial of combined treatment of endoscopic mucosal resection and chemoradiotherapy for clinical stage I esophageal carcinoma: Japan Clinical Oncology Group Study JCOG0508. Jpn J Clin Oncol. 2009;39(10):686-9.

14 Muto M, Hironaka S, Nakane M, Boku N, Ohtsu A, Yoshida S. Association of multiple Lugol-voiding lesions with synchronous and metachronous esophageal squamous cell carcinoma in patients with head and neck cancer. Gastrointest Endosc. 2002;56(4):517-21.

15 Fukuhara T, Hiyama T, Tanaka S, Oka S, Yoshihara M, Arihiro K, et al. Natural course of Lugol-voiding lesions in patients with superficial esophageal squamous cell carcinoma. Digestion. 2010;82(1):60-5. 
16 Minashi K, Nihei K, Mizusawa J, Takizawa K, Yano T, Ezoe Y, et al. Efficacy of endoscopic resection and selective chemoradiotherapy for stage I esophageal squamous cell carcinoma. Gastroenterology. 2019;157(2):382-e3.

17 Shimizu Y, Kato M, Yamamoto J, Nakagawa S, Tsukagoshi H, Fujita M, et al. EMR combined with chemoradiotherapy: a novel treatment for superficial esophageal squamouscell carcinoma. Gastrointest Endosc. 2004; 59(2):199-204.

18 Kawaguchi G, Sasamoto R, Abe E, Ohta A, Sato H, Tanaka K, et al. The effectiveness of endoscopic submucosal dissection followed by chemoradiotherapy for superficial esophageal cancer. Radiat Oncol. 2015;10:31.

19 Yoshimizu S, Yoshio T, Ishiyama A, Tsuchida T, Horiuchi Y, Omae M, et al. Long-term out- comes of combined endoscopic resection and chemoradiotherapy for esophageal squamous cell carcinoma with submucosal invasion. Dig Liver Dis. 2018;50(8):833-8.

20 Katada C, Yokoyama T, Yano T, Oda I, Shimizu Y, Takemura K, et al. Association between the findings of metachronous secondary primary malignancies and the number of Lugol-voiding lesions. Dis Esophagus. 2020 Feb 12; doz110.

21 Muto M, Hitomi Y, Ohtsu A, Ebihara S, Yoshida S, Esumi H, et al. Association of aldehyde dehydrogenase 2 gene polymorphism with multiple oesophageal dysplasia in head and neck cancer patients. Gut. 2000;47(2): 256-61.

22 Kagemoto K, Urabe Y, Miwata T, Oka S, Ochi $\mathrm{H}$, Kitadai $\mathrm{Y}$, et al. ADH1B and ALDH2 are associated with metachronous SCC after endoscopic submucosal dissection of esophageal squamous cell carcinoma. Cancer Med. 2016;5(7):1397-404.

23 Abiko S, Shimizu Y, Miyamoto S, Ishikawa M, Matsuda K, Tsuda M, et al. Risk assessment of metachronous squamous cell carcinoma after endoscopic resection for esophageal carcinoma based on the genetic polymorphisms of alcoholdehydrogense-1B aldehyde dehydrogenase-2: temperance reduces the risk. J Gastroenterol. 2018;53(10):1120-30.

24 Morita M, Kumashiro R, Kubo N, Nakashima Y, Yoshida R, Yoshinaga K, et al. Alcohol drinking, cigarette smoking, and the development of squamous cell carcinoma of the esophagus: epidemiology, clinical findings, and prevention. Int J Clin Oncol. 2010;15(2):126-34. 